\title{
CONE COMPLEXES AND PL TRANSVERSALITY
}

\author{
BY \\ CLINT MCCRORY(1)
}

\begin{abstract}
A definition of PL transversality is given, using the orderreversing duality on partially ordered sets. David Stone's theory of stratified polyhedra is thereby simplified; in particular, the symmetry of blocktransversality is proved. Also, polyhedra satisfying Poincaré duality are characterized geometrically.
\end{abstract}

The purpose of this paper is to develop a simple theory of transversality for polyhedra in piecewise linear manifolds. Our main tool is a canonical geometric duality for "structured" cone complexes. Cone complexes have also been used by M. Cohen [7] and E. Akin [2] to study simplicial maps. The rigid geometry provided by a structured cone complex on a polyhedron is analogous to a Riemannian metric on a smooth manifold (as manifested by its relation to duality and transversality). In fact, structured cone complexes are equivalent to van Kampen's combinatorial "star complexes" [10] (1929).

PL transversality has been studied by M. A. Armstrong and E. C. Zeeman ([4], [3]), C. P. Rourke and B. J. Sanderson ([15] , [17], [18]), and D. Stone [22]. The stumbling block of Stone's stratified blocktransversality theory was the question of whether transversality of polyhedra in a manifold is symmetric. Furthermore, symmetry of blocktransversality easily implies that Stone's definition is equivalent to Rourke and Sanderson's "mocktransversality" for polyhedrayielding a unified and versatile theory.

Our analysis of transversality stems from its close relation to Poincaré duality-in fact our definition echoes Lefschetz' classical (1930) definition of transversality of cycles in a combinatorial manifold [12]. If $X$ is a closed PL manifold, and $C$ is a cell complex on $X$, a choice of cone structures for the cells of $\mathrm{C}$ determines a dual cell complex $\mathrm{C}^{*}$. We define polyhedra $P$ and $Q$ to be transverse in $X$ if there is such a structured cell complex $C$ on $X$ with $P$ a subcomplex of $C$ and $Q$ a subcomplex of $C^{*}$. This definition is symmetric, and our main result is that it is equivalent to Stone's definition.

Received by the editors February 8, 1974. $55 \mathrm{C} 05$.

AMS (MOS) subject classifications (1970). Primary 57C99, 54E20; Secondary 06A10,

Key words and phrases. Piecewise linear transversality, blocktransversality, cone complex, poset, piecewise linear stratification, intrinsic dimension, Poincaré duality.

(1) Supported in part by NSF grant GP $36418 \times 1$. 
In $\S 1$, we discuss the geometry of partially ordered sets, which are in effect "abstract" cone complexes. $\$ 2$ sets out the theory of cone complexes, structures, and geometric duality. In $\S 3$, we analyze Stone's PL stratifications in terms of cone complexes. $\$ 4$ contains our definition of transversality and the proof that it is equivalent to Stone's definition. Finally, in $\S 5$, we consider questions of transversality when the ambient space $X$ is not a manifold, where the "obstruction" to transversality is seen to be due precisely to the failure of Poincaré duality in $X$.

I would like to thank David Stone not only for teaching me about stratifications, but also for the chats which contained the seeds of many of the ideas presented here.

1. Preliminaries. A polyhedron is a topological space with a maximal family of piecewise-linearly related (locally finite) triangulations. By a triangulation of a polyhedron we will always mean a triangulation from this family (in other words, a PL triangulation). A homeomorphism $X \cong Y$ of polyhedra will always mean a PL homeomorphism.

If $X$ is a polyhedron and $x \in X, 1 \mathrm{k}(x ; X)$ denotes the link of $x$ in any triangulation of $X$ with $x$ a vertex. $1 \mathrm{k}(x ; X)$ is well defined up to PL homeomorphism, and a neighborhood of $x$ in $X$ (st $(x ; X)$, the star of $x)$ is homeomorphic to the cone on $\operatorname{lk}(x ; X)$. Thus polyhedra are locally conical, a fact which can be used as their definition [19].

As a combinatorial prelude to our discussion of the geometry of polyhedra, we discuss products, joins, and duality for partially ordered sets, simplifying van Kampen's theory of star complexes ([10], [20, §66]).

Recall that an (abstract) simplicial complex $K$ is a set of nonempty finite subsets of a given set $V(K)$ (the set of vertexes of $K$ ) such that $\{v\} \in K$ for all $v$ $\in V(K)$, and if $\sigma \in K$ and $\tau \subseteq \sigma$, then $\tau \in K$. The elements of $K$ are called its simplexes, and if $\tau \subseteq \sigma$ one says $\tau$ is a face of $\sigma$. (We will always assume that $K$ is locally finite: if $\tau \in K$, there are only finitely many $\sigma \in K$ with $\tau \subseteq \sigma$.) If $K$ and $L$ are simplicial complexes, a simplicial map $f: K \rightarrow L$ is a function $V(f)$ : $V(K) \rightarrow V(L)$ such that if $\sigma \in K$, then $V(f)(\sigma) \in L$. Simplicial complexes and simplicial maps form a category, which we will denote by $(S)$.

There is a functor from $(S)$ to the category of polyhedra and piecewise linear maps which assigns to a simplicial complex $K$ (or a simplicial map $f$ ) its geometric realization $|K|$ (or $|f|$ ). $|K|$ can be defined as a union of standard euclidean simplexes, with identifications according to the face relation in $K$.

A poset (partially ordered set) $(A, \leqslant)$ is a set $A$ with a reflexive, transitive, antisymmetric relation $\leqslant$. That is to say, if $a, b, c \in A$, 
(i) $a \leqslant a$,

(ii) $a \leqslant b$ and $b \leqslant c$ implies $a \leqslant c$, and

(iii) $a \leqslant b$ and $b \leqslant a$ implies $a=b$.

If $a \leqslant b$ and $a \neq b$, one writes $a<b$. (We will always assume that $A$ is locally finite: if $a \in A$, there are only finitely many $b \in A$ with $a<b$ or $b<a$.) If $(A, \leqslant)$ and $(B, \leqslant)$ are posets, a function $f: A \rightarrow B$ is isotone (order preserving) if $a \leqslant b$ in $A$ implies $f(a) \leqslant f(b)$ in $B$. Partially ordered sets and order preserving functions form a category $(P)$. (For further discussion, see [6, Chapter I] .)

There are canonical functors $i:(S) \rightarrow(P)$ and $\eta:(P) \rightarrow(S)$ defined as follows: $i(K)=(K, \subseteq)$, the set $K$ with its face relation. $i$ can be thought of as an inclusion of categories, but $(S)$ is not a full subcategory of $(P)$. (Not all functions between simplicial complexes which preserve the face relation arise from simplicial maps.)

$\eta(A, \leqslant)$ is the nerve of $(A, \leqslant)$. The vertexes of $\eta(A, \leqslant)$ are the elements of $A$, and its simplexes are all finite subsets of $A$ of the form $\left\{a_{0}, a_{1}, \cdots, a_{i}\right\}$ with $a_{0}<a_{1}<\cdots<a_{i}$. Such a set will be written $\left\langle a_{0}, a_{1}, \cdots, a_{i}\right\rangle$ to emphasize that it is ordered.

Neither $i$ nor $\eta$ is an equivalence of categories. For example, if $A=\{a, b\}$ with $a<b$, there is no complex $K$ with $i(K) \cong(A, \leqslant)$. On the other hand, if $K$ is the boundary of the standard 2-simplex, there is no $(A, \preccurlyeq)$ with $\eta(A, \leqslant) \cong K$. Notice, however, that if $K$ is any simplicial complex, $\eta i(K)=K^{\prime}$, the first derived complex of $K$. This is the geometrical explanation for the close relation between $(S)$ and $(P)$.

From now on, we will omit the ordering from our notation, and write $A=$ $(A, \leqslant)$.

The most striking property of posets not shared by simplicial complexes is the canonical duality between a poset $A$ and the same set with the reverse ordering, which we will denote $\hat{A}$. Clearly $A=\hat{\hat{A}}$, and $\eta(\hat{A})=\eta(A)$. The geometric significance of this duality will unfold in $\S 2$-for the moment we will be content with its formal properties.

If $A$ and $B$ are posets, their product $A \times B$ is the set of ordered pairs $(a, b), a \in A, b \in B$, with $\left(a_{1}, b_{1}\right) \leqslant\left(a_{2}, b_{2}\right)$ if $a_{1} \leqslant b_{1}$ and $a_{2} \leqslant b_{2}$. There is no natural definition of the product of two simplicial complexes-one must order the vertexes. However, $\eta(A)$ and $\eta(B)$ are simplicial complexes with vertex orderings (total on each simplex) inherited from $A$ and $B$, and if we form the simplicial complex $\eta(A) \times \eta(B)$ using these orderings, $\eta(A) \times \eta(B)=\eta(A \times B)$. Thus the product of posets corresponds to the usual geometric notion.

Proposition 1.1. If $A$ and $B$ are partially ordered sets, $(A \times B) \hat{=}=\hat{A} \times \hat{B}$. 
The proof is trivial.

The join of two finite posets $A$ and $B$ can be defined in two ways:

(i) $A * B$ is the disjoint union of $A$ and $B$ with their given partial orders, and with $a>b$ for all $a \in A, b \in B$.

(ii) (van Kampen) $A \circledast B$ is the disjoint union of $A, A \times B$, and $B$, with the partial order given by the orders on these sets, plus the condition that $a_{1}<$ $\left(a_{2}, b\right)$ if $a_{1} \leqslant a_{2}$, and $b_{1}<\left(a, b_{2}\right)$ if $b_{1} \leqslant b_{2}$.

Recall that the join $K * L$ of two finite simplicial complexes has for its vertex set the disjoint union of $V(K)$ and $V(L)$, with simplexes $\sigma, \tau$, and $\sigma * \tau=$ $\sigma \cup \tau, \sigma \in K, \tau \in L$. The geometric realization $|K * L|$ is the space of all line segments from $|K|$ to $|L|$.

Clearly $i(K * L)=i(K) \circledast i(L)$, which is a subdivision of $i(K) * i(L)$. On the other hand, $\eta(A * B)=\eta(A) * \eta(B)$, which has $\eta(A) \circledast \eta(B)$ as a subdivision.

If $A$ is a finite poset, the cone on $A$ is the $*$-join of a point with $A, c A=$ $\{c\} * A$. The following result is due to van Kampen [10, p. 12]

PROPOSITION 1.2. Let $A$ and $B$ be finite posets. There is a canonical isomorphism $c(\hat{A}) \times c(\hat{B}) \cong c(A * B)^{\wedge}$.

Proof. The elements of $c(\hat{A}) \times c(\hat{B})$ are of four types:

(1) $(c, c)$,

(2) $(a, c), a \in A$,

(3) $(c, b), b \in B$,

(4) $(a, b), a \in A, b \in B$.

These correspond to the four types of elements in $c(A * B)^{\wedge}$ :

(1) $c$,

(2) $a \in A$,

(3) $b \in B$,

(4) $(a, b), a \in A, b \in B$.

It is easy to check that this correspondence is order preserving. Q.E.D.

Several standard polyhedral facts (which we will need subsequently) are corollaries of Proposition 1.2. If $X$ is a compact polyhedron, let $c X$ denote the cone on $X$.

COROLlaRY 1.3. If $X$ and $Y$ are compact polyhedra, $c X \times c Y \cong c(X * Y)$, and $X * Y \cong(X \times c Y) \cup(c X \times Y)$, where the union is along $X \times Y$.

COROLlary 1.4. If $X$ and $Y$ are polyhedra, $x \in X$, and $y \in Y$,

$$
\operatorname{lk}((x, y) ; X \times Y) \cong \operatorname{lk}(x ; X) * \operatorname{lk}(y ; Y) .
$$

Let $I$ denote the unit interval $\{x \in \mathbf{R}, 0 \leqslant x \leqslant 1\}$. 
COROLlaRY 1.5. If $X$ is a polyhedron, and $x \in X, 1 \mathrm{k}((x, 0) ; X \times I) \cong$ $c(\operatorname{lk}(x ; X))$.

2. Cone complexes. We adopt the following useful notation, due to Stallings [21, §1.6]. If $S$ is a set of sets, and $A$ is a set, $A \sqrt{ } S$ means $A$ is a union of elements of $S$.

Definition. A cone complex $C$ on a polyhedron $X$ is a locally finite covering of $X$ by compact subpolyhedra, together with a subpolyhedron $\partial \alpha$ of each element $\alpha$ of $C$ such that

(i) for each $\alpha \in C, \partial \alpha \sqrt{ } C$,

(ii) if $\alpha$ and $\beta$ are distinct elements of $C, \stackrel{\circ}{\alpha} \cap \stackrel{\circ}{\beta}$ is empty, where $\stackrel{\circ}{\alpha}$ $=\alpha \backslash \partial \alpha$,

(iii) for each $\alpha$ in $C$, there is a (PL) homeomorphism $\alpha \cong c(\partial \alpha)$ rel $\partial \alpha$.

We will write $X=|C|$, and refer to the elements of $C$ simply as the cones of $\mathrm{C}$.

Note that (i) and (ii) imply that, if $\alpha$ and $\beta$ are cones of $C$, then $\alpha \cap \beta \sqrt{ } C$. It should also be emphasized that $\partial \alpha$ is not necessarily the topological boundary of $\alpha$.

EXAMPLES. (1) A triangulation, or more generally a cellulation of a polyhedron, is a cone complex. We will suppress triangulating homeomorphisms, and think of a triangulation $K$ of $X$ as a collection of closed simplexes covering $X$. By a cell complex, we will always mean a cone complex in which each cone $\alpha$ is a ball, with $\partial \alpha$ its boundary sphere.

(2) The classical "dual complex" to a triangulation $K$ of $X$ is a cone complex, comprising the "dual cones" $\sigma^{*}$ to simplexes $\sigma$ of $K$, where $\sigma^{*}=$ $\bigcap_{\tau \leqslant \sigma}$ st $\left(\tau, K^{\prime}\right)$ in the first derived complex $K^{\prime}$, where $\underline{\tau}$ denotes the barycenter of $\tau$.

All of the terminology of cell complexes generalizes to cone complexes. Let $C$ be a cone complex on $X$. If $\alpha, \beta$ are cones of $C$, and $\alpha \subset \partial \alpha$, we say $\alpha$ is a (proper) face of $\beta$, and write $\alpha<\beta$. The height of a cone $\alpha$ of $C$ is the maximum integer $i$ such that there is a sequence $\alpha_{0}<\alpha_{1}<\cdots<\alpha_{i}=\alpha$ in $C$. The $i$ skeleton $C_{i}$ of $C$ consists of all cones of $C$ of height $\leqslant i$. $C_{i}$ covers an $i$-dimensional subpolyhedron of $X$ (by induction on $i$. Note that height $\alpha=0 \Leftrightarrow \partial \alpha=\varnothing$ $\Leftrightarrow \alpha$ is a point). Dually, the depth of a cone $\alpha$ of $C$ is the maximum integer $j$ such that there is a sequence of cones $\alpha=\alpha_{0}<\alpha_{1}<\cdots<\alpha_{j}$ in $C$. The cones of depth 0 are called principal.

A subcomplex $D \subset C$ is a subset such that $\alpha<\beta$ in $C$ and $\beta \in D$ implies $\alpha$ $\in D$. If $A \subset X$ is a union of cones of $C$, we write $A \sqrt{ } C$, and if $A$ is the union of all the cones of the subcomplex $D$ we write $|D|=A$, or $D=C \mid A$. (We will say that $A$ is a subcomplex of $C$, when no confusion can occur.) $A$ 
subdivision $B$ of $C$ is a cone complex with $|B|=|C|$ such that $\alpha \in C$ implies $\alpha \sqrt{ } B$.

Cone complexes were introduced by Akin [2], following ideas of Cohen [7]. He also introduced the concept of "structuring" a cone complex to study its geometry.

Let $C$ be a cone complex on $X$. If $\alpha$ is a cone of $C$, a homeomorphism $f_{\alpha}: \alpha \cong c(\partial \alpha)$ rel $\partial \alpha$ is called a structure for $\alpha$. The apex (cone point) $\underline{\alpha}$ of $\alpha$ in this structure is $f_{\alpha}^{-1}(c)$. A structure for $C$ is a choice of structures for each cone in $C$. By Alexander's trick any two structures for a cone $\alpha$ of $C$ are isotopic, keeping $\partial \alpha$ fixed. Furthermore, this isotopy can be extended to an ambient isotopy of $X=|C|$ by conewise extension over each (structured) cone $\beta$ with $\alpha<\beta$. Thus a structure for $C$ is "unique up to isotopy." However, we will be concerned with the rigid geometry defined by a fixed structure.

EXAMPLES. (1) A triangulation has a canonical "barycentric" structure, for a simplex $\sigma$ is the join of its barycenter $\underline{\sigma}$ with its boundary.

(2) An arbitrary cell complex does not have a preferred structure. By a structured cell complex, we will mean a cell complex structured as a cone complex. Note that by our convention above the cone points must lie in the interiors of the cells.

(3) If $K$ is a triangulation, the dual cone complex $K^{*}$ has a canonical structure, for the dual cone $\sigma^{*}$ is $\underline{\sigma} * \operatorname{link}^{\prime}(\sigma)$, where $\operatorname{link}^{\prime}(\sigma)=\left\{\left\langle\underline{\tau}_{0}, \cdots, \underline{\tau}_{i}\right\rangle\right.$ $\left.\in K^{\prime}, \sigma<\tau_{0}\right\}$.

(4) Let $A$ be a (locally finite) poset, and let $X=|\eta(A)|$. Define a cone complex $C$ on $X$ with one cone $\alpha$ for each element $a \in A, \alpha=\mid \eta\{b \in A$, $b \leqslant a\} \mid$. Not only is $C$ clearly a cone complex, but $C$ also has a canonical structure, since $\eta\{b \in A, b \leqslant a\}$ is a simplicial cone with apex $a$. The following proposition says that structured cone complexes are precisely such "geometric realizations" of posets.

Proposition 2.1. A structured cone complex $C$ on $X$ has a canonical derived subdivision $C^{\prime}$, which is a triangulation of $X$ with vertexes the apexes of cones of $\mathrm{C}$. As an abstract simplicial complex, $\mathrm{C}^{\prime}$ is the nerve of poset $\mathrm{C}$ (with its face ordering).

Proof. $C^{\prime}$ is constructed by induction up the skeletons of $C$. If $\alpha \in C$ has height $0, \alpha$ is a point, so $\alpha$ is a vertex of $C^{\prime}$. If $\alpha$ has positive height, let $f_{\alpha}: \alpha \cong c(\partial \alpha)($ rel $\partial \alpha)$ be the given structure for $\alpha$. Let $C^{\prime} \mid \alpha$ be the subdivision induced by $f_{\alpha}^{-1}$ from $c * C^{\prime} \mid \partial \alpha$, the simplicial cone on the inductively defined subdivision of $\partial \alpha$. Thus the simplexes in $C^{\prime}$ are of the form $\left\langle\underline{\alpha}_{0}, \cdots, \underline{\alpha}_{i}\right\rangle$, 
$\alpha_{0}<\cdots<\alpha_{i}$ in $C$, so abstractly, $C^{\prime}$ is just the nerve of $C$.

Duality.

TheOREM 2.2. Let $\mathrm{C}$ be a structured cone complex on $X$. There is a canonical dual structured cone complex $C^{*}$ on $X$, with $\left(C^{*}\right)^{*}=C$, and $\left(C^{*}\right)^{\prime}=C^{\prime}$. There is a bijection $\alpha \leftrightarrow \alpha^{*}$ between the cones of $C$ and $C^{*}$ such that $\alpha<\beta \Leftrightarrow$ $\beta^{*}<\alpha^{*}, \underline{\alpha}^{*}=\underline{\alpha}$, and $\mid$ st $\left(\alpha, C^{\prime}\right) \mid$ is homeomorphic to $\alpha \times \alpha^{*}$.

Proof. The existence of $C^{*}$ is a formal consequence of Proposition 2.1 and $\S 1$. In concrete terms, $C^{*}$ is constructed from $C$ just as is the dual complex to a triangulation. If $\alpha \in C, \alpha$ is the union of all simplexes $\left\langle\underline{\alpha}_{0}, \cdots, \underline{\alpha}_{i}\right\rangle$ in $C^{\prime}$ with $\alpha_{i} \leqslant \alpha . \alpha^{*}$ is the union of all simplexes $\left\langle\underline{\alpha}_{0}, \cdots, \underline{\alpha}_{i}\right\rangle$ in $C^{\prime}$ with $\alpha \leqslant \alpha_{0}$. Thus $C^{\prime} \mid \alpha^{*}$ is the simplicial join of $\underline{\alpha}$ with $\operatorname{lk}^{\prime}(\alpha)=\left\{\left\langle\underline{\alpha}_{0}, \cdots, \underline{\alpha}_{i}\right\rangle \in C^{\prime}, \alpha<\alpha_{0}\right\}$, so $\alpha^{*}$ is a structured cone. $\partial\left(\alpha^{*}\right)=\left|\mathrm{kk}^{\prime}(\alpha)\right|=\bigcup_{\alpha<\beta^{*}} \beta^{*}$, so $\partial\left(\alpha^{*}\right)$ is a union of cones of $C^{*}$. Clearly $\alpha^{*} \cap \beta^{*}=\bigcup_{\alpha, \beta<\gamma} \gamma^{*}$, so $\left(\alpha^{*}\right)_{\circ} \cap\left(\beta^{*}\right)^{\circ}=\varnothing$ if $\alpha^{*} \neq \beta^{*}$.

It remains to show $\mid$ st $\left(\alpha, C^{\prime}\right) \mid \cong \alpha \times \alpha^{*}$. Now

$$
\begin{aligned}
\mid \text { st }\left(\underline{\alpha}, C^{\prime}\right) \mid & =\left|\underline{\alpha} * \operatorname{lk}\left(\alpha, C^{\prime}\right)\right|=\left|\underline{\alpha} *\left(C^{\prime} \mid \partial \alpha\right) *\right| \mathrm{k}^{\prime}(\alpha) \mid \\
& =c\left(\partial \alpha *|| \mathrm{k}^{\prime}(\alpha) \mid\right) \cong c(\partial \alpha) \times c|| \mathrm{k}^{\prime}(\alpha) \mid \text { by Corollary } 1.4 \\
& =\alpha \times \alpha^{*} .
\end{aligned}
$$

EXAMPLE. Let $K$ be a triangulation of the closed $n$-manifold $M$. Define a map $M \rightarrow \mathbf{R}$ by $f(\underline{\sigma})=\operatorname{dim} \sigma$ for $\sigma \in K$, and let $f$ be linear on each simplex of $K^{\prime}$. Then $f$ is a PL Morse function [11], and the critical points of $f$ are the barycenters of simplexes of $K$. The handle decomposition of $M$ associated to $f$ is just $K$ thickened up. The dual Morse function $-f$ (got by "turning $M$ upside-down") has as its handle decomposition a thickening of $K^{*}$, the classical dual cell complex to $K$. More generally, if $C$ is a structured cone complex on a polyhedron $X$, we can define a "Morse function" $f: X \rightarrow \mathbf{R}$ by $f(\alpha)=$ height $\alpha$ for each cone $\alpha$ of $C$, and extend $f$ conically over the cones of $C$ (by induction on their height) using the given structure. (Thus if $\sigma$ is a simplex of $C^{\prime}$, the values of $f$ on the vertexes of $\sigma$ are distinct. Such functions do enjoy some of the algebraic properties of a classical Morse function-cf. [5] .) The cones of $C$ can be recovered.as the "descending polyhedra" of the critical points, and the linear cone structures can be "pulled back" from $\mathbf{R}$. In this way, the dual Morse function $g=-f$ is associated with the dual structured cone complex $C^{*}$.

\section{Products.}

Proposition 2.3. If $C$ is a cone complex on $X$ and $D$ is a cone complex on $Y$, then $C \times D=\{\alpha \times \beta, \alpha \in C, \beta \in D\}$ is a cone complex on $X \times Y$. Structures for $C$ and $D$ determine a structure for $C \times D$, and $(C \times D)^{*}=C^{*} \times D^{*}$. 
Proof. This follows immediately from Propositions 2.1 and 1.1

Restriction. Following Akin [2], we now analyze the restriction of a cone complex to a subpolyhedron. Let $C$ be a cone complex on $X$ and let $A$ be a subpolyhedron of $X$. C induces a cone complex on $A$ if, for each cone $\alpha$ in $C$ such that $\stackrel{\circ}{\alpha} \cap A \neq \varnothing$,

$$
\alpha \cap A \cong c(\partial \alpha \cap A) \operatorname{rel}(\partial \alpha \cap A) .
$$

Then we define $C \mid A=\{\alpha \cap A, \alpha \in C$ and $\stackrel{\circ}{\alpha} \cap A \neq \varnothing\}$, with $\partial(\alpha \cap A)=$ $(\partial \alpha) \cap A$.

Suppose further that $C$ is structured, and the structure on the cone $\alpha$ restricts to a structure on $\alpha \cap A$ for each $\alpha$ with $\stackrel{\circ}{\alpha} \cap A \neq \varnothing$. (That is, if $f_{a}: \alpha \cong$ $c *(\partial \alpha)$ rel $\partial \alpha$ is the given structure on $\alpha, f_{a} \mid \alpha \cap \partial \alpha: \alpha \cap \partial \alpha \cong c *(\partial \alpha \cap A)$ rel $\partial \alpha \cap A$.) Then we say that $C$ induces a structured cone complex on $A$. (See [2] for a discussion of extending structures.)

LEMmA 2.4. The structured cone complex $C$ on $X$ induces a structured cone complex on the subpolyhedron $A \subset X$ if and only if $A$ is a full subcomplex of $\mathrm{C}^{\prime}$.

Proof. Induction on the dimension of $A$ (i.e., the height of $C \mid A$ ) yields the result easily.

Proposition 2.5. Let $\mathrm{C}$ be a structured cone complex on $X$. If $\mathrm{C}$ induces a structured cone complex on $A \subset X$, then so does $C^{*}$, and $(C \mid A)^{*}=C^{*} \mid A$.

Proof. Since $\left(C^{*}\right)^{\prime}=C^{\prime}$, Lemma 2.4 implies that $C$ induces a structured cone complex on $A$ if and only if $C^{*}$ does. Clearly, $(C \mid A)^{\prime}=C^{\prime} \mid A$, so if $\stackrel{\circ}{\alpha} \cap A \neq \varnothing$, the dual of $\alpha \cap A$ in $(C \mid A)^{*}$ is covered by the simplexes $\left\langle\underline{\alpha}_{0}, \cdots\right.$, $\left.\underline{\alpha}_{i}\right\rangle$ in $C^{\prime}$ lying in $A$ with $\alpha<\alpha_{0}$, and so equals $\alpha^{*} \cap A$. Q.E.D.

Subdivision. Let $C$ be a cone complex, and $D$ a subcomplex. $D$ is a full subcomplex of $C$ if $\alpha, \beta \in D$ and $\gamma \in C$, with $\alpha, \beta \leqslant \gamma$, implies there exists $\delta \in D$ with $\alpha, \beta \leqslant \delta \leqslant \gamma$. (In other words, each $\gamma \in C$ has at most one maximal face in $D$. This is equivalent to the usual definition when $C$ and $D$ are simplicial complexes.) A subdivision $B$ of $C$ is a full subdivision if every subcomplex of $C$ is subdivided by a full subcomplex of $B$.

LEMMA 2.6. Any structured cone complex C has a structured subdivision B such that

(i) $B$ is a full subdivision of $C$,

(ii) $B^{*}$ is a full subdivision of $C^{*}$,

(iii) $B^{\prime}$ is a full subdivision of $C^{\prime}$. 
Proof. Recall that if $A$ is a poset, the set $\{c\} \circledast A$ is defined with a partial ordering so that $\eta(\{c\} \circledast A)$ is a subdivision of $\eta(c A)(\S 1)$. Thus, by the equivalence of structured cone complexes and posets (Proposition 2.1), if $A$ is a structured cone complex we can form a structured cone complex $c \circledast A$, with $|c \circledast A|$ $=c|A|$.

We will prove the lemma by induction on the height of $C$, using the following observation: If $A$ is a cone complex and $D$ is a full subcomplex, then $c \circledast D$ is a full subcomplex of $c \circledast A$.

If height $C=0$, the lemma is trivial. So suppose height $C=n$, and the lemma is true for complexes of height $<n$. Thus we assume $B|| C_{n-1} \mid$ has been defined, so we just need to define $B \mid \gamma$ for each principal cone $\gamma$ of $C$, given $B \mid \partial \gamma$. Let $K=(\gamma \circledast(B \mid \partial \gamma))^{\prime}$, a triangulation of $\gamma$ (depending on the given structure of $\gamma$ ). Let the cones of $B \mid \gamma$ be the following sets (not the cones of $\underline{\gamma} \circledast(B \mid \partial \gamma)$ ):

(1) $|\mathrm{st}(\gamma ; K)|$,

(2) $(\underline{\gamma} * \beta) \cap|\mathrm{lk}(\underline{\gamma} ; K)|$, for each $\beta \in B \mid \partial \gamma$,

(3) $(\underline{\gamma} * \beta)-\mid$ st $(\gamma ; K) \mid$, for each $\beta \in B \mid \partial \gamma$,

(4) all $\beta \in B \mid \partial \gamma$.

We now define the cone structures for the sets (1)-(3). The cone (1) is homeomorphic to $\gamma$ by "pseudo-radial projection," which sends the cones (2) of its boundary to the corresponding cones (4) of $B \mid \partial \gamma$. The cones (1) and (2) are structured by these identifications. Now each cone (3) is canonically homeomorphic to $\beta \times I$, being the stellar neighborhood of $\beta$ in $\underline{\gamma} * \beta$. (Thus all the cones (3) form a collar of $\partial \gamma$ in $\gamma$.) Let the structure on a cone (3) correspond to the product of the given structure on $\beta$ with the usual structure $I=\{1 / 2\}$ * $\{0,1\}$. Now $B \mid \gamma$ is clearly a structured cone complex subdivision of $\mathrm{Cl} \gamma$ (i.e., $\gamma$ and its faces).

It remains to verify (i), (ii), (iii) of the lemma for $\mathrm{B} \mid \gamma, \mathrm{Cl} \gamma$ (which imply (i), (ii), (iii) for $B, C$ ).

(i) We must show that each subcomplex $D$ of $\mathrm{Cl} \gamma$ intersects each cone $\alpha$ of $B \mid \gamma$ in at most one maximal face of $\alpha$. This is clear for $\alpha$ of type (1) or (2), since $\alpha \subset \stackrel{\circ}{\gamma}$, and it is true for type (4) by inductive hypothesis. If $\alpha$ is of type (3), $\alpha \cap \partial \gamma=\beta \in B \mid \partial \gamma$, so since $D$ intersects $B$ in at most one maximal face, $D$ intersects $\alpha$ in at most one maximal face.

(ii) First note that if $\alpha \in B \mid \gamma$, each cone of $C^{*} \mid \gamma$ intersects $\alpha$ in a subcone of $\alpha$ (with respect to the given structure on $\alpha$ ). This is clear for $\alpha$ of type (1), (2), or (4); and it is true for type (3) because $C^{*} \mid \gamma$ respects the canonical collar of $\partial \gamma$ in $\gamma$. Thus $C^{*}$ induces a cone complex $C^{*} \mid \alpha$ on $\alpha$. As nonstructured cone complexes, $C^{*} \mid \alpha=\underline{\alpha} \circledast\left(C^{*} \mid \partial \alpha\right)$. Now to show (ii), it suffices to show that $B^{*} \mid \alpha$ is a full subdivision of $C^{*} \mid \alpha$ for each principal cone $\alpha \in B \mid \gamma$ (i.e., $\alpha$ is type (1) or (3)). 
(This is because of Proposition 2.5.) This condition holds for (1) because $C^{*} \mid \alpha$ corresponds to $C^{*} \mid \gamma=\underline{\gamma} *\left(C^{*} \mid \partial \gamma\right)$ under the homeomorphism $\alpha \cong \gamma, B^{*} \mid \alpha$ corresponds to $\gamma \circledast\left(B^{*} \mid \partial \gamma\right)$, and $B^{*} \mid \partial \gamma$ is a full subdivision of $C^{*} \mid \partial \gamma$ by inductive hypothesis. (Here we apply our preliminary observation that coning preserves fullness.) The condition holds for type (3) by induction on height $\alpha$, since $B^{*} \mid \alpha=\underline{\alpha} \circledast\left(B^{*} \mid \partial \alpha\right)$ and $C^{*} \mid \alpha=\underline{\alpha} \circledast\left(C^{*} \mid \partial \alpha\right)$ (as nonstructured complexes).

(iii) The argument is the same as for (ii)-just replace $B^{*}, C^{*}$ by $B^{\prime}, C^{\prime}$.

REMARK. The inductive construction of the subdivision $B$ is canonical, given the structure on $C$.

Intrinsic dimension. To analyze how the cones of a complex intersect the "singularities" of the underlying polyhedron, we invoke the theory of intrinsic dimension, developed by Armstrong [3]. For a complete discussion of intrinsic dimension, with geometric applications, see [1].

If $x$ is a point in the subpolyhedron $A$ of $X$, the intrinsic dimension $d(x ; X, A)$ of $(X, A)$ at $x$ is the largest integer $i$ such that there is a (PL) triangulation of $(X, A)$ with $x$ in the interior of an $i$-simplex. Equivalently, $d(x ; X, A)$ is the largest integer $i$ such that $\mathrm{lk}(x ; X, A)$ is an $i$-fold suspension. $d(x ; X, \varnothing)$ $=d(x ; X)$ is the intrinsic dimension of $X$ at $x$. Clearly $d(x ; X, A) \leqslant d(x ; X)$, and $d(x ; X, A) \leqslant d(x ; A)$. $\in \stackrel{\circ}{\alpha}$,

Theorem 2.7. Let $\mathrm{C}$ be a cone complex on $X$, and let $\alpha \in C$. Then if $x$

(i) $d(x ; X, \alpha)=d(x ; \alpha)$,

(ii) $d(x ; X) \leqslant d(x ; \alpha)+$ depth $\alpha$.

Remarks. Property (i) says that $\alpha$ is locally unknotted in $X$, in the sense of Akin [1]. Combining (i) and (ii), we have $d(x ; \alpha) \leqslant d(x ; X) \leqslant d(x ; \alpha)+$ depth $\alpha$ for all $x \in \stackrel{\circ}{\alpha}$.

In fact, this theorem is true for Akin's "general complexes" [2], in which property (iii) of a cone complex $(\alpha \cong c(\partial \alpha)$ rel $\partial \alpha)$ is weakened to $\partial \alpha$ is collared in $\alpha$. This is because each facet of a general complex has a little "transverse star." This has been exploited by Cohen and Sullivan [9] for manifold complexes, and clarified by Rourke and Sanderson [18].

PRoof. Choose a structure for C. By the proof of Theorem 2.2, if $x \in \stackrel{\circ}{\alpha}$, $\alpha \in C$,

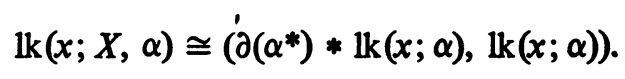

(i) We have $d(x ; X, \alpha) \leqslant d(x ; \alpha)$ a priori, so we must show $d(x ; X, \alpha) \geqslant$ $d(x ; \alpha)$. By the second characterization of intrinsic dimension, this says 
$\mathrm{lk}(x ; X, \alpha)$ desuspends at least as much as $\mathrm{k}(x ; \alpha)$, which is true by (*).

(ii) We must show that $1 \mathrm{k}(x ; X)$ desuspends at most $q$ more times than $\operatorname{lk}(x ; \alpha)$, where $q=\operatorname{depth} \alpha$. This follows from (*) by results of Morton. More precisely, suppose $\operatorname{lk}(x ; X) \cong S^{k} * A$ and $\operatorname{lk}(x ; \alpha) \cong S^{l} * B$, where $A$ and $B$ are not suspensions. Then by $(*)$,

$$
S^{k} * A \cong \partial\left(\alpha^{*}\right) * S^{l} * B
$$

Write $\partial\left(\alpha^{*}\right) \cong S^{m} * C$, where $C$ is not a suspension. Then

$$
S^{k} * A \cong S^{m+l+1} * B * C .
$$

Now by $\left[14\right.$, Theorem 1], $k=m+l+1$. But $m \leqslant \operatorname{dim} \partial\left(\alpha^{*}\right)=q-1$, so $k \leqslant l+q$. Q.E.D.

The intrinsic $i$-skeleton of a polyhedron $X$ is $\{x \in X, d(x ; X) \leqslant i\}$, or the intersection of the $i$-skeletons of all triangulations of $X$. The intrinsic $i$-stratum of $X$ is $\{x \in X ; d(x ; X)=i\}$. (ii) says that if $q=\operatorname{depth} \alpha$, the intersection of $\stackrel{\circ}{\alpha}$ with the intrinsic $i$-skeleton of $X$ contains the intrinsic $(i-q)$-skeleton of $\stackrel{\circ}{\alpha}$.

Definition. The cone complex $C$ on $X$ is transverse if for each $\alpha \in C$, $d(x ; X)=d(x ; \alpha)+\operatorname{depth} \alpha$ for all $x \in \stackrel{\circ}{\alpha}$.

Corollary 2.8. Let $\mathrm{C}$ be a structured cone complex. C is transverse if and only if $\mathrm{C}^{*}$ is a cell complex.

Proof. By the proof of Theorem 2.7, $d(x ; X)=d(x ; \alpha)+$ depth $\alpha$ if and only if $\partial\left(\alpha^{*}\right)$ is a sphere, i.e., $\alpha^{*}$ is a cell.

LEMMA 2.9. A cone complex is a cell complex if and only if its principal cones are cells.

Proof. A cone $(\alpha, \partial \alpha)$ is a cell if and only if it is a manifold with boundary. But if $\beta<\alpha$, and depth $\beta=$ depth $\alpha+1, \alpha$ is a manifold implies $\beta$ is, since $\partial \alpha$ is collared in $\alpha$ (apply Corollary 1.5). Thus if the principal cones of a complex are cells, all its cones are cells, by induction on their depth.

Proposition 2.10. Let $\mathrm{C}$ be a cone complex on $X$. C is a cell complex if and only if for each cone $\alpha, \stackrel{\circ}{\alpha}$ is contained in an intrinsic stratum of $X$.

Proof. If $\alpha$ is a principal cone of $C, d(x ; \alpha)=d(x ; X)$ for $x \in \stackrel{\circ}{\alpha}$, so if $\alpha$ lies in an intrinsic stratum, $\alpha$ is a cell. Thus $C$ is a cell complex by Lemma 2.9. Conversely, if $C$ is a cell complex, and $\alpha \in C$, for each pair of points $x, y \in \stackrel{\circ}{\alpha}$ there is an isotopy of $\alpha$ which takes $x$ to $y$, keeping $\partial \alpha$ fixed. This isotopy extends conically over each $\beta>\alpha$ to an ambient isotopy of $X$, so $d(x ; X)=d(y ; X)$. 
REMARK. Our original approach to cone complexes and duality was through "cone coordinates," using cone rays in each cone. (Cone coordinates can be expressed in terms of barycentric coordinates in the derived complex.) Such a tack is plagued by "standard mistakes," and conceals the combinatorial simplicity of cone complexes. However, it does seem to have a certain geometrical affinity with the smooth category (smooth manifolds and Thom's stratified sets).

3. Stratified polyhedra. In this section we discuss the relation between cone complexes and Stone's PL stratification theory. This not only enhances the geometry of cone complexes, but also sheds some light on stratifications. First we summarize the definition of a stratified polyhedron-for a complete discussion, see Stone's notes [22].

Let $X$ be a polyhedron. A filtration $X=X_{n} \supset X_{n-1} \supset \cdots \supset X_{0}$ by subpolyhedra is called a variety of $X$ if for each point $x$ in $X$, say $x \in X_{i}-X_{i-1}$, there are an integer $k$ and a compact polyhedron $L$ such that a neighborhood $N$ of $x$ in $X$ is homeomorphic to $D^{k} \times c L$, with $X_{i} \cap N$ corresponding to $D^{k} \times\{c\}$. Furthermore, for each $j>i, X_{j} \cap N$ corresponds to $D^{k} \times c L_{j}$, for some subpolyhedron $L_{j} \subset L$.

Clearly, if $X_{i}=X_{i}-X_{i-1}$ is nonempty, it is a disjoint union of manifolds of various dimensions $k . X_{i}$ is called the $i$ th stratum of the given variety. The definition essentially says that each stratum $X_{i}$ is "equisingular"; that is, $X$ has locally constant "normal geometry" along $X_{i}$. The filtration of a polyhedron by the skeletons of a triangulation, or by its intrinsic skeletons, is a variety. A Whitney stratification of a real analytic variety has a canonical PL structure in which it is a PL variety [23].

REMARK. Stone's varieties are slightly more general; he refers to the above class as "skeletal varieties" [22, Chapter $1, \S 2]$. His definition facilitates the discussion of "polyhedra with boundary," which are necessary for his treatment of cobordism with singularities.

A regular neighborhood system for the variety $\left(X_{n}, \cdots, X_{0}\right)$ is a collection of subpolyhedra $N_{i j}$ of $X, n \geqslant i \geqslant j \geqslant 0$, such that for each $k, X_{k}=$ $\bigcup_{k \geqslant j} N_{k j}, X_{k} \cap N_{i j}=N_{k j}$ for $i \geqslant k$, and $N_{k j}$ is a regular neighborhood of $N_{j j}$ in $\operatorname{cl}\left(X_{k} \backslash \cup_{j>l} N_{k l}\right)$ which meets the boundary regularly. (For an elaboration of this definition, and some appealing illustrations, see [22, Chapter 1, §3].) In short, each stratum is equipped with a regular neighborhood in each of the higher strata incident to it, and these neighborhoods fit together neatly.

A stratification of a polyhedron $X$ with respect to a variety filtration $\left(X_{n}, \cdots, X_{0}\right)$ is a regular neighborhood system $\left\{N_{i j}\right\}$, plus a block bundle 
structure $\xi_{i j}$ on each $N_{i j}$, such that all the blocks intersect nicely (see [22, Chapter $1, \S 4]$ ). More precisely, $\xi_{i j}$ is a block bundle with total space $N_{i j}$, base $N_{i j}$, and fiber a cone (possibly different cones for different components of $N_{j j}$ ). Thus a typical block $\beta$ is homeomorphic to $D^{k} \times c L$ for some compact polyhedron $L$. Therefore $\beta \cong c\left(S^{k-1} * L\right)$, so each block is a cone. (The boundary of $\beta$ as a cone is $\partial \beta \cup \beta^{*}$ in Stone's notation $[22$, p. 30].) Thus the blocks of the bundles $\xi_{n j}, n \geqslant j \geqslant 0$, define a partition of $X=X_{n}=\bigcup_{j} N_{n j}$ into cones. It follows from Stone's definition [22, p. 35, axiom bs4] that this partition is in fact a cone complex, which we will call the cone complex associated to the given stratification of $X$.

THEOREM 3.1. Let $\left(X_{n}, \cdots, X_{0}\right)$ be a variety filtration of the polyhedron $X=X_{n}$, and let $C$ be a cone complex on $X . C$ is the cone complex associated to a stratification of $X$ with respect to the variety $\left(X_{n}, \cdots, X_{0}\right)$ if and only if there is a structure for $C$ such that $X_{i}$ is a full subcomplex of $C^{*}$ for each $i$.

Remark. By Proposition 2.10, it follows that $C^{*}$ is a cell complex, since $d(x ; X)$ is locally constant for $x \in X_{i}$ by equisingularity.

Corollary 3.2. A cone complex $\mathrm{C}$ on a polyhedron $X$ is associated to some stratification of $X$ with respect to the intrinsic variety of $X$ if and only if $C$ is transverse and satisfies the following intersection property:

(*) If $\alpha \in C$, let $d(\alpha)=\min \{d(x ; X), x \in \alpha\}$. Then if $\alpha, \beta, \gamma \in C$, with $\gamma$ a principal cone of $\mathrm{C} \mid \alpha \cap \beta, d(\gamma)=\max \{d(\alpha), d(\beta)\}$.

Proof. If $C$ is transverse, $C^{*}$ is a cell complex for any structure on $C$, so the intrinsic skeletons of $X$ are subcomplexes of $C^{*}$ (by Proposition 2.10). Furthermore, since $d(x ; X)$ is constant along cone rays of a cone $\alpha$, except at the apex $\underline{\alpha}, d(\alpha)=d(\alpha ; X)=d\left(\alpha^{*} ; X\right)$, so $d(\alpha)=i \Leftrightarrow \alpha^{*} \subset X_{i}$. Thus property (*) is dual to the statement that each intrinsic skeleton of $X$ is covered by a full subcomplex of $C^{*}$. Therefore the corollary follows from the theorem.

Proof of theorem. First we show that if $C$ is the cone complex associated to a stratification of $X$ with respect to $\left(X_{n}, \cdots, X_{0}\right)$, then $C$ can be structured so that each $X_{i}$ is a full subcomplex of $C^{*}$. Let $\left\{\xi_{i j}, n \geqslant i \geqslant\right.$ $j \geqslant 0$ \} be the cone blockbundles comprising the given stratification. By definition, the cones of $C$ are the blocks of the bundles $\xi_{n j}, n \geqslant j \geqslant 0$. According to Stone's definition [22, p. 35, axioms bsl, bs2], for each $j,\left(\xi_{n j}, \cdots, \xi_{(j+1) j}\right)$ is a blockbundle "flag" over the cell complex $\xi_{j j}$, and the total space of $\xi_{i j}$ is $N_{i j}$, where $\left\{N_{i j}\right\}$ is a regular neighborhood system for $\left(X_{n}, \cdots, X_{0}\right)$. Thus, if $\beta$ is a block of $\xi_{n j}$ (i.e., a cone of $C$ ), $\beta \cap X_{i}=\beta \cap N_{i j}$ is the block of $\xi_{i j}$ lying in $\beta$. 
Thus the definition of a flag [22, p. 33] implies that each cone $\beta$ of $C$ can be structured so that $\beta \cap X_{i}$ is a structured subcone for each $i$. Define a structure for $C$ by choosing such a structure for each cone $\beta$. Now $X_{i} \sqrt{ } C^{*}$ if and only if $\alpha^{*}, \beta^{*} \in C^{*}, \alpha^{*}<\beta^{*}, \beta^{*} \subset X_{i}$ implies $\alpha^{*} \subset X_{i}$. Since the structure on $C$ induces a structured cone complex on $X_{i}$ by definition, $X_{i}$ is a full subcomplex of $C^{\prime}=\left(C^{*}\right)^{\prime}$ (Lemma 2.4). Thus to show $X_{i} \sqrt{ } C$, we need only show that if $\alpha, \beta \in C, \alpha>\beta, \underline{\beta} \in X_{i}$ implies $\underline{\alpha} \in X_{i}$. Now $\underline{\beta} \in X_{i}$ means $\beta$ is a block of $\xi_{p i}$ for some $p$. Suppose $\alpha$ is a block of $\xi_{q j}$. Since $\alpha<\beta$, Stone's definition (axiom bs4) implies $j=i$, i.e., $\underline{\alpha} \in X_{i}$. By the same token, $X_{i}$ is a full subcomplex of $C^{*} \Leftrightarrow \alpha, \beta, \gamma \in C, \gamma$ a principal cone of $C \mid \alpha \cap \beta$, and $\underline{\alpha}, \underline{\beta} \in X_{i}$, implies $\underline{\gamma} \in X_{i}$. Say $\alpha \in \xi_{p i}, \beta \in \xi_{q i}$. Then $\gamma \in \xi_{r i}$ for some $r$, again by Stone's definition (axiom bs4), so $\chi \in X_{i}$. This proves the first half of the theorem.

For the converse we must show that, given a variety $\left(X_{n}, \cdots, X_{0}\right)$ and a structured cell complex $B$ on $X=X_{n}$ with each $X_{i}$ a full subcomplex of $B$, a stratification for $X$ with respect to $\left(X_{n}, \cdots, X_{0}\right)$ can be constructed out of the cones of $B^{*}$. We simply repeat Stone's construction [22, pp. 43-49]. He starts with a simplicial complex $B$ such that each $X_{i}$ is a full subcomplex $B_{i}$, and constructs a stratification using the dual cones in $B_{i}$ to simplexes of $B$, for all $n \geqslant i \geqslant 0$. Let $D\left(\sigma, B_{i}\right)$ denote the dual of $\sigma$ in $B_{i}$. By Proposition 2.5, $D\left(\sigma, B_{i}\right)=\sigma^{*} \cap B_{i}$, so his construction only uses the cones of $B^{*}$ and their intersections with the given variety filtrations of $X$. (Since we are not concerned with "boundaries" of polyhedra, we can ignore the extra cones in his construction needed for boundaries.) It is easy to see that the only place where he uses the fact that $B$ is simplicial, rather than cellular, is in checking that certain subpolyhedra are regular neighborhoods (e.g., that the neighborhoods built out of the cones of $B^{*}$ form a regular neighborhood system for $X$ ). Here he invokes Cohen's definition of a regular neighborhood as a stellar neighborhood in the first derived complex of some (full) triangulation. Therefore, to transfer Stone's arguments to cell complexes, we just need the following result.

Lemma 3.3. Let $\mathrm{C}$ be a structured cell complex on $X$, and let $Y=|D|$, where $D$ is a full subcomplex of $C$. Then $\left|N\left(D^{\prime}, C^{\prime}\right)\right|=\mid\left\{\sigma \in C^{\prime}\right.$; there exist $\left.\omega \in D^{\prime}, \tau \in C^{\prime}, \sigma \leqslant \tau, \omega \leqslant \tau\right\} \mid$ is a regular neighborhood of $Y$ in $X$.

Proof. This is an easy consequence of Cohen's stellar neighborhood theorem $[8$, p. 204] .

REMARK. As a corollary of this proof, Stone's axiom bs 3 for a stratified polyhedron $X$ [22, p. 35] is a consequence of his other three axioms, if the underlying filtration of $X$ is a variety. This is because bs 3 was not used to derive 
the characteristic properties of the cone complex associated to a stratification. Furthermore, it is not logically necessary to state that the neighborhoods $N_{i j}$ (the total spaces of the bundles $\xi_{i j}$ ) are regular neighborhoods-this is a consequence of bsl and bs4, and Cohen's stellar neighborhood theorem.

Corollary 3.4. Let $\mathrm{C}_{1}$ and $\mathrm{C}_{2}$ be transverse cone complexes on $X$. There are transverse subdivisions $D_{1}, D_{2}$ of $C_{1}, C_{2}$ respectively, and an isotopy $h_{t}$ of $X$ such that $h_{1}: D_{1} \cong D_{2}$ (i.e., $h_{1}$ maps each cone of $D_{1}$ onto a cone of $D_{2}$, and $h_{1}(\partial \alpha)=\partial h_{1}(\alpha)$ for all $\left.\alpha \in D_{1}\right)$.

Proof. This follows from Stone's uniqueness theorem [22, p. 58]. It can also be proved directly using stellar subdivisions.

4. Transversality of polyhedra in manifolds. In this section and the next, we show how cone complexes can be used to unify the theory of PL transversality, as developed by Armstrong and Zeeman, Rourke and Sanderson, and Stone. As a corollary of this analysis, we show that blocktransversality of polyhedra in a manifold is symmetric, which settles an important question of Stone.

We begin with a discussion of cone complexes on PL manifolds.

PROPOSITION 4.1. If $X$ is a polyhedron, the following are equivalent.

(a) $X$ is a PL manifold without boundary.

(b) Any cone complex on $X$ is a cell complex.

(c) Any cone complex on $X$ is transverse.

(d) There is a structured cell complex $\mathrm{C}$ on $X$ such that $\mathrm{C}^{*}$ is a cell complex.

Proof. (a) $\Rightarrow$ (b) by Proposition 2.10. (b) $\Leftrightarrow$ (c) because $C$ is transverse $\Leftrightarrow \mathrm{C}^{*}$ is cellular (Corollary 2.8). Clearly (b) $\Rightarrow$ (d). (d) $\Rightarrow$ (a) because if $x \in X$, say $x \in \stackrel{\circ}{\sigma}, \sigma \in C, 1 \mathrm{k}(x ; X) \cong \partial \sigma * \partial\left(\sigma^{*}\right)$, so if $C$ and $C^{*}$ are cellular, $\mathrm{lk}(x ; X)$ is a sphere for all $x \in X$.

If $X$ is a manifold with boundary, the situation is not much more complicated. Let $C$ be a cone complex on $X$. Then if $\sigma$ is a cone of $C, \partial \sigma$ is either a sphere or a ball. If $\partial \sigma$ is a sphere, either $\stackrel{\circ}{\sigma} \subset(X \backslash \partial X)$ or $\stackrel{\circ}{\sigma} \subset \partial X$. If $\partial \sigma$ is a ball, $\stackrel{\circ}{\sigma} \cap(X \backslash \partial X) \neq \varnothing$ and $\stackrel{\circ}{\sigma} \cap \partial X \neq \varnothing$. By definition, $C$ is a cell complex if and only if $\partial \sigma$ is a sphere for all $\sigma \in \mathcal{C}$. Thus it is clear that $C$ is a cell complex if and only if $\partial X \sqrt{ } \mathrm{C}$. If $\mathrm{C}$ is a structured cell complex, and $\partial X$ is a full subcomplex of $C, \mid\left\{\sigma^{*} \in C^{*}, \sigma \in C^{*}, \sigma \cap \partial X \neq \varnothing\right\}$ is a collar of $\partial X$ in $X$.

Definition. Let $P$ and $Q$ be subpolyhedra of the manifold $X . P$ is transverse to $Q$, written $P \pitchfork Q$, if there is a structured cone complex $C$ on $X$ such that $Q \sqrt{ } C$ and $P \sqrt{ } C^{*}$.

Note that $P \pitchfork Q \Leftrightarrow Q \pitchfork P$, since $\left(C^{*}\right)^{*}=\mathrm{C}$. By Proposition 4.1, if $X$ has no 
boundary then $C$ and. $C^{*}$ are cell complexes.

REMARK. It is interesting to note that a triangulation $K$ of a (closed) manifold $X$ and its dual cell complex $K^{*}$ do not have the symmetry of a structured cell complex and its dual. Specifically, if the (locally flat) submanifold $Y$ of $X$ is a subcomplex of $K^{*}$, the normal bundle of $Y$ is a fiber bundle (i.e., its structural group can be reduced from $\widetilde{\mathrm{PL}}$ to $\mathrm{PL}$ ). Since normal blockbundles are not always fibered [16], there may exist submanifolds $Y$ of $X$ which cannot be subcomplexes of the dual to any triangulation of $X$-so the transversality theorem cannot be true if we use a triangulation in our definition of transversality. This observation led Armstrong and Zeeman to introduce the concept of "transimpliciality" (see [4, p. 433]).

Proper subpolyhedra. To simplify our discussion of transversality in a manifold $X$, we will assume that subpolyhedra intersect $\partial X$ transversely. We say the subpolyhedron $P$ of $X$ is proper if $(\partial X, P \cap \partial X)$ is collared in $(X, P)$.

Remark. ( $(\partial X, P \cap \partial X)$ is collared in $(X, P)$ if and only if $(\partial X, P \cap \partial X)$ is locally collared in $(X, P)$ (cf. [1, p. 455]). It is easy to see that if $P$ is a subpolyhedron of the manifold $X, P$ is proper if and only if $P \pitchfork \partial X$. We will actually need a stronger result:

Lemma 4.2. Let $P$ be a proper subpolyhedron of the manifold $X$. Given any structured cone complex $C$ on $X$ with $P \sqrt{ } C^{*}$, there is a structured subdivision $B$ of $C$ such that $B$ is a cell complex and $P \sqrt{ } B^{*}$.

Proof. We construct $B$ by altering $C$ slightly. The cones $\alpha \in C$ with $\partial \alpha$ a sphere are left unchanged. If $\partial \alpha$ is a ball, we replace $\alpha$ by $\widetilde{\alpha}$, with $\widetilde{\alpha}=\alpha$ as a subset of $X$, and $\partial \widetilde{\alpha}=\partial \alpha \cup(\alpha \cap \partial X)$. We also add the cell $\beta=\alpha \cap \partial X$ to fill up $\partial(\widetilde{\alpha})$. The structure on $\beta$ is induced by the given structure on $\alpha$. Now $\widetilde{\alpha} \cong$ $(\alpha \cap \partial X) \times I$, so $\widetilde{\alpha}$ can be structured as a cell, but we must choose this structure to respect $P \cap \widetilde{\alpha}$. More precisely, if $P \cap \stackrel{\circ}{\alpha} \neq \varnothing$, there is a homeomorphism $f:(\alpha, P \cap \alpha) \cong(\alpha \cap \partial X, P \cap \alpha \cap \partial X) \times[0,1]$ with $f(x)=(x, 0)$ for $x \in \alpha \cap$ $\partial X$, since $(\alpha, P \cap \alpha)=$ st $(\alpha ; X, P),(\alpha \cap \partial X, P \cap \alpha \cap \partial X)=$ st $(\alpha ; \partial X, P \cap \partial X)$, and $(\partial X, P \cap \partial X)$ is collared in $(X, P)$. If $g: \alpha \cong c(\partial \alpha)$ is the given structure for $\alpha$, and $h: I \cong c\{0,1\}$ is the standard structure for $I,[(g \mid \alpha \cap \partial X) \times h] \circ f$ defines a structure for $\widetilde{\alpha}$. By this construction, $P$ intersects each (open) structured cone of $B$ in a subcone, so (by Lemma 2.4) to show that $P \sqrt{ } B^{*}$, it is enough to check that if $\beta, \gamma \in B$, with $\beta<\gamma$, then $P \cap \beta \neq \varnothing$ implies $P \cap \stackrel{\circ}{\gamma} \neq \varnothing$. But this is clear from our construction, since $P \sqrt{ } C^{*}$.

Corollary 4.3. If $P$ and $Q$ are subpolyhedra of the manifold $X$, with $P$ proper, then $P \pitchfork Q$ if and only if there is a structured cell complex $C$ on $X$ such that $Q \sqrt{ } C$ and $P \sqrt{ } C^{*}$. 
Blocktransversality. Stone [22, Chapter 4] has defined blocktransversality of polyhedra in manifolds generalizing Rourke and Sanderson's concept of blocktransversality for submanifolds [15]. If $P$ is blocktransverse to $Q$, we will write $P \perp Q$.

THEOREM 4.4. If $P$ and $Q$ are proper subpolyhedra of the manifold $X$, $P \perp Q$ if and only if $P \pitchfork Q$.

Corollary 4.5 (SYmmetry of blockTRANSVERSAlity). If $\boldsymbol{P}$ and $Q$ are proper subpolyhedra of the manifold $X, P \perp Q \Leftrightarrow Q \perp P$.

REMARK. The definition of blocktransversality we will use is slightly less general than Stone's, since his definition of variety is broader (cf. §3). In fact, the theorem is true for arbitrary subpolyhedra $P$ and $Q$ with his more general definition of $P \perp Q$, and the proof is only a bit more involved.

Proof. Stone defines $P \perp Q$ if $Q$ has a "stratified normal blockbundle" in $X$ which $P$ intersects in a union of blocks. A stratified normal blockbundle for $Q$ in $X$, or an " $(n+1, n)$-stratification" in Stone's terminology, is almost the same as a stratification of $X$ with respect to the filtration $\left(X_{n+1}, X_{n}, \cdots, X_{0}\right)$, where $X=X_{n+1}, Q=X_{n}$, and $\left(X_{n}, \cdots, X_{0}\right)$ is the intrinsic variety of $Q$ in $X$ (i.e., $\operatorname{dim} Q=n$, and $X_{i}=\{x \in Q, d(x ; X, Q) \leqslant i\}$ for $\left.i \leqslant n\right)$. The only difference is that an $(n+1, n)$-stratification of $Q$ in $X$ does not include the cellulation $\xi_{(n+1)(n+1)}$ of $N_{(n+1)(n+1)}=\operatorname{cl}\left(X \backslash \bigcup_{n \geqslant j} N_{(n+1) j}\right)$ (cf. $\S 3$ above and [22, Chapter 1, §4]). Since $N=\bigcup_{n \geqslant j} N_{(n+1) j}$ is a regular neighborhood of $Q$ in $X$, it is fruitful to think of an $(n+1, n)$-stratification of $Q$ in $X$ as a normal blockbundle $\xi / Q$ with total space $N$. (This is the basic theme of Stone's work.) In these terms, $P \perp Q$ means that there exists $\xi / Q$ in $X$ such that $P \cap N$ is a union of blocks of the cone blockbundles $\xi_{(n+1) j}$ for $0 \leqslant j \leqslant n$. We now proceed to the proof of the theorem.

$P \pitchfork Q \Rightarrow P \perp Q$ : Since $P$ is proper, there is a structured cell complex $C$ on $X$ with $Q \sqrt{ } C$ and $P \sqrt{ } C^{*}$ (Corollary 4.3). By Lemma 2.6, there is a structured subdivision $B$ of $C$ such that $B^{*}$ is a full subdivision of $C^{*}$. Since $C$ is a cell complex, the intrinsic strata of $Q$ in $X$ are unions of open cells of $C$. Thus, by Theorem 3.1, $\mathrm{C}^{*}$ is the cone complex associated to some stratification $\left\{\xi_{i j}\right\}$ of $X$ with respect to the intrinsic variety $\left(X_{n+1}, X_{n}, \cdots, X_{0}\right)$ of $Q=X_{n}$ in $X=X_{n+1}$. Throwing away the blocks of $\xi_{(n+1)(n+1)}$, we obtain a normal blockbundle $\xi / Q$ in $X$ which $P$ intersects in blocks. $\left(P \sqrt{ } B^{*}\right.$, whose cones are blocks of $\xi_{(n+1) j}, 0 \leqslant j \leqslant n+1$.)

$P \perp Q \Rightarrow P \pitchfork Q$ : Let $\xi / Q$ be a normal bundle with total space $N$ which $P$ intersects in blocks. Let $\dot{K}$ be a triangulation of $\overline{X \backslash N}$ such that $\overline{P \backslash N}$ and all 
the sets $\beta \cap \partial N, \beta$ a block of $\xi$, are subcomplexes of $K$. Let $C$ be the cone complex on $X$ consisting of all the blocks $\beta$ of the bundles $\xi_{(n+1) j}, 0 \leqslant j \leqslant$ $n$, plus the simplexes of $K$. By the proof of Theorem 3.1 (namely, by the definition of a blockbundle flag), $C$ has a structure such that each intrinsic stratum of $Q$ in $X$ is a union of open cones of $C^{*}$. In particular, $Q \sqrt{ } C^{*}$ and $P \sqrt{ } \mathrm{C}$ (by construction), so $P \oplus Q$. Q.E.D.

REMARK. This argument can clearly be made with respect to any stratification of $Q$ in $X$.

Mocktransversality. The following definition of transversality arises from Rourke and Sanderson's elegant description of geometric homology theories and duality [18].

If $C$ is a cell complex, and $P$ is a subpolyhedron of $|C|$, we will say that $P$ is transverse to $C$ if $(\partial \alpha, P \cap \partial \alpha)$ is collared in $(\alpha, P \cap \alpha)$ for each cell $\alpha$ of $C$.

DEFinition. If $P$ and $Q$ are subpolyhedra of the manifold $X, P$ is mocktransverse to $Q$ if there is a cell complex $C$ on $X$ such that $Q \sqrt{ } C$ and $P$ is transverse to $C$.

THEOREM 4.6. If $P$ and $Q$ are proper subpolyhedra of the manifold $X, P$ is mocktransverse to $Q$ if and only if $P \pitchfork Q$.

Proof. If $P \pitchfork Q$, then by Lemma 4.2 there is a structured cell complex $C$ on $X$ with $Q \sqrt{ } C$ and $P \sqrt{ } C *$. By Proposition 2.5, $P \cap \alpha$ is a structured subcone of $\alpha$ for each $\alpha \in C$, so $(\partial \alpha, P \cap \partial \alpha)$ is collared in $(\alpha, P \cap \alpha)$.

Conversely, suppose $P$ is mocktransverse to $Q$, with respect to the cellulation $C$ of $X$. We will define a structured cellular subdivision $D$ of $C$ such that $P \sqrt{ } D^{*}$. We construct $D \mid \alpha$ for each $\alpha \in C$, by induction on height $\alpha$. (Note that $(P \cap \alpha) \sqrt{ } D^{*} \mid \alpha$ for all $\alpha$ implies $P \sqrt{ } D^{*}$.) Now a cell $\alpha$ is a manifold with boundary $\partial \alpha$, and $P \cap \alpha$ is a proper subpolyhedron by mocktransversality. We assume inductively that $D \mid \partial \alpha$ has been defined so that $(P \cap \partial \alpha) \sqrt{ } D^{*} \mid \partial \alpha$. By using a collar, $D^{*} \mid \partial \alpha$ can be extended to a transverse cone complex $B$ on $\alpha$ with $(P \cap \alpha) \sqrt{ } B$. Let $D \mid \alpha=B^{*}$. This completes the inductive step.

REMARK. In [18], Rourke and Sanderson point out that $P$ is mocktransverse to $Q$ if and only if $Q$ is blocktransverse to $P$. This observation was a germinal point in the development of my definition of transversality.

Note that Theorem 4.6 is not true if $P$ is not proper, because the proof shows that if $P$ is mocktransverse to $Q$ in $X, P$ is a proper subpolyhedron of $X$.

It is not hard to see that if $P$ and $Q$ are subpolyhedra of the manifold $X$, $P$ is mocktransverse to $Q$ if and only if $P$ is transimplicial to $Q$, in the sense of Armstrong and Zeeman ([4], [3]). The hallmark of these definitions, as well as ours, is that they are "global"-a cellulation of the entire ambient manifold is used. 
In fact, Rourke and Sanderson [17] have shown that $P$ and $Q$ "locally" transverse in the sense of Armstrong [3] does not imply $P \perp Q$, even if $P$ and $Q$ are locally flat submanifolds.

The fault with local PL transversality is that the relative transversality theorem is false, even for submanifolds (Hudson, unpublished). That is, there exist (locally flat) proper submanifolds $P$ and $Q$ of a manifold $X$, such that $P \cap \partial X$ and $Q \cap \partial X$ are locally transverse in $\partial X$, but there is no isotopy of $X$ keeping $\partial X$ fixed which moves $P$ locally transverse to $Q$. However, the relative transversality theorem is true for blocktransversality ([15], [22]). Rourke and Sanderson have found a quite simple proof of the relative mocktransversality theorem for maps [18], which easily adapts to a proof of the following theorem. By Theorem 4.4, Stone's transversality theorem is a corollary.

THEOREM 4.7. Let $P$ and $Q$ be proper subpolyhedra of the compact manifold $X$, with $(P \cap \partial X) \pitchfork(Q \cap \partial X)$ in $\partial X$. There is an arbitrarily small ambient isotopy $h_{t}$ of $X$ such that $h_{t} \mid \partial X$ is the identity for all $t$, and $h_{1}(P) \pitchfork Q$.

REMARKS. (1) Some restriction on the way $P$ and $Q$ meet $\partial X$ is essential if the isotopy is to keep $\partial X$ fixed. This is because if $P_{i}=\{x \in P, d(x ; X, P) \leqslant$ $i$, then $P \pitchfork Q$ implies $\left(P_{i} \cap \partial X\right) \pitchfork\left(Q_{j} \cap \partial X\right)$ in $\partial X$ for all $i, j$, so this condition must be true at the outset (cf. [22, pp. 86-87]). However, if $X$ is the 3-disc $\left\{\left(x_{1}, x_{2}, x_{3}\right),-1 \leqslant x_{1} \leqslant 1,-1 \leqslant x_{2} \leqslant 1,0 \leqslant x_{3} \leqslant 1\right\}$, and $P=\left\{x \in X, x_{3}=\right.$ $\left.\left|x_{1}\right|\right\}, Q=\left\{x \in X, x_{3}=\left|x_{2}\right|\right\}$, then $\left(P_{i} \cap \partial X\right) \pitchfork\left(Q_{i} \cap \partial X\right)$, but no isotopy of $X$ keeping $\partial X$ fixed can move $P$ transverse to $Q$ ( $P$ nor $Q$ is proper).

(2) The proof can be made with respect to any variety filtration of $X$ (see [22, Chapter $1, \S 2]$, for the definition), so Stone's transversality theorem is indeed a corollary.

\section{Transversality to a stratification.}

Definition. Let $\left(X_{n}, \cdots, X_{0}\right)$ be a variety filtration of the polyhedron $X=X_{n}$, and let $P$ be a subpolyhedron of $X . P$ is transverse to $\left(X_{n}, \cdots, X_{0}\right)$, written $P \pitchfork\left(X_{n}, \cdots, X_{0}\right)$, if there is a structured cone complex $C$ on $X$ such that $X_{i} \sqrt{ } C$ for each $i$ and $P \sqrt{ } C^{*}$.

Remark. By Proposition 2.10, any cone complex $C$ with $X_{i} \sqrt{C}$ for all $i$ is a cell complex.

By the proof of Theorem 3.1,P巾 $\left(X_{n}, \cdots, X_{0}\right)$ if and only if there is a stratification $\xi=\left\{\xi_{i j}\right\}$ of $X$ with respect to $\left(X_{n}, \cdots, X_{0}\right)$ such that $P$ intersects each bundle $\xi_{n j}$ (and hence each bundle $\xi_{i j}$ ) in a union of blocks. In other words, $P$ is blocktransverse to each stratum.

If $X$ is a manifold and $\left(X_{n}, \cdots, X_{0}\right)$ is the intrinsic variety of the 
subpolyhedron $Q=X_{n-1}$ in $X, P \pitchfork\left(X_{n}, \cdots, X_{0}\right)$ just says $P \pitchfork Q$ (and $P$ is proper).

Recall that if $\left(X_{n}, \cdots, X_{0}\right)$ is the intrinsic variety of $X$ and $C$ is a cellulation of $X$, then $X_{i} \sqrt{ } C$ for each $i$ (Proposition 2.10). Thus if $P$ is transverse to any variety of $X, P$ is transverse to the intrinsic variety. Rourke and Sanderson's mocktransversality technique yields the following generalization of Theorem 4.7.

THEOREM 5.1. Let $P$ be a subpolyhedron of $X$ which is transverse to the intrinsic variety of $X$. If $\left(X_{n}, \cdots, X_{0}\right)$ is any variety of $X$, there is an arbitrarily small ambient isotopy $h_{t}$ of $X$ such that $h_{1}(P) \pitchfork\left(X_{n}, \cdots, X_{0}\right)$.

For example, we can take $\left(X_{n}, \cdots, X_{0}\right)$ to be the intrinsic variety of a subpolyhedron $Q$ in $X$. Thus if $P$ is transverse to the "singularities" of $X, P$ can be isotoped transverse to any polyhedron $Q$ in $X$. But not every subpolyhedron can be made transverse to the singularities of $X$ in the first place.

EXAMPLE. Let $X=\left(S^{1} \times S^{1}\right) /\left(S^{1} \times\{p\}\right), p \in S^{1}$, the pinched torus, and let $P$ be the image of $\{p\} \times S^{1}$ under the identification map. The intrinsic 0 -stratum of $X$ is just the singular point $x_{0}$. If $C$ is a structured cell complex on $X, x_{0}$ must be a vertex of $C$, and its dual is thus a principal cone of $C^{*}$, so any 1 dimensional subcomplex of $C^{*}$ misses $x_{0}$. However, no isotopy of $P$ in $X$ can move $P$ off $x_{0}$, so $P$ cannot be made transverse to the intrinsic stratification of $X$. In fact, if we think of $P$ as a mod 2 cycle, $P$ is not homologous to a cycle which misses $x_{0}$.

The following result, which is based on [13], characterizes the "obstruction" to moving a cycle in $X$ (via homology) transverse to the intrinsic stratification.

THEOREM 5.2. Let $X$ be a normal polyhedral n-circuit with fundamental class $[X]$. A homology class $z \in H_{s}(X)$ is represented by a PL cycle whose support is transverse to the intrinsic stratification of $X$ if and only if $z=[X] \cap y$ for some cohomology class $y \in H^{n-s}(X)$.

REMARKS. A normal $n$-circuit ("orientable polyhedron") is an $n$-dimensional polyhedron $X$ such that the restriction homomorphism $H_{n}(X) \rightarrow$ $H_{n}(X, X \backslash\{x\})$ is an isomorphism for all $x \in X . H_{*}(X), H^{*}(X)$ denote singular homology and cohomology with integral coefficients. (The theorem is true mutatis mutandis for arbitrary coefficients.) $\cap$ is cap product.

Proof. We use the following formula for cap product [13]. Let $K$ be a structured cell complex on $X$, and let $C_{*}(K), C^{*}(K)$ be the oriented cellular chains and cochains on $K$. (We assume that the cells of $K$ have been arbitrarily oriented.) Cap product is induced by the pairing 


$$
C_{p}(K) \otimes C^{q}(K) \rightarrow C_{p-q}\left(K^{\prime}\right)
$$

given by

$$
\alpha_{p} \cap \beta_{q}=\sum \pm\left\langle\underline{\alpha}_{p}, \underline{\gamma}_{p-1}, \cdots, \underline{\gamma}_{q+1}, \underline{\beta}_{q}\right\rangle .
$$

Here $\alpha_{p}$ is an elementary $p$-chain (oriented $p$-cell), $\beta_{q}$ is an elementary $q$-cochain, and the sum is taken over all sequences of cells $\alpha_{p}>\gamma_{p-1}>\cdots>\gamma_{q+1}>\beta_{q}$ with $\operatorname{dim} \gamma_{i}=i$. The coefficient $\epsilon\left(\alpha_{p}, \gamma_{p-1}, \cdots, \gamma_{q+1}, \beta_{q}\right)= \pm 1$ is defined as follows. Let $[\alpha, \gamma]= \pm 1$ denote the incidence number of the oriented cells $\alpha$ and $\gamma$. Then

$$
\epsilon\left(\alpha_{p}, \gamma_{p-1}, \cdots, \gamma_{q+1}, \beta_{q}\right)=\left[\alpha_{p}, \gamma_{p-1}\right]\left[\gamma_{p-1}, \gamma_{p-2}\right] \cdots\left[\gamma_{q+1}, \beta_{q}\right] .
$$

Now suppose that the orientations for the $n$-cells of $K$ were chosen coherently, so that their sum $\Sigma_{i} \gamma_{n}^{i}$ represents the fundamental class. Then

$$
\left(\sum_{i} \gamma_{n}^{i}\right) \cap \beta_{n-s}=\sum \pm\left\langle\underline{\gamma}_{n}, \underline{\gamma}_{n-1}, \cdots, \underline{\beta}_{n-s}\right\rangle \text {, }
$$

the sum of all the $s$-simplexes of $K^{\prime} \mid\left(\beta_{n-s}\right)^{*}$, with appropriate orientations. Thus $z=[X] \cap y$ implies that $z$ is represented by a simplicial cycle in $K^{\prime}$ whose support is a union of cones of $K^{*}$, and hence is transverse to the intrinsic stratification of $X$.

Conversely, suppose $z \in H_{s}(X)$ is represented by a PL cycle $c$ whose support is transverse to the intrinsic stratification of $X$, i.e., there is a structured cell complex $K$ on $X$ such that (support $c$ ) $\sqrt{ } K^{*}$. It is clear that by subdividing $K^{\prime}$ so that $c$ is simplicial, and then "amalgamating" $c$ on each simplex of $K^{\prime}$, we can assume that $c$ is a simplicial cycle on $K^{\prime}$. Thus it remains to show that an integer $\mu(\beta)$ can be assigned to each $(n-s)$-cell $\beta$ in $K$ with $\beta^{*} \subset$ support $c$, so that $\left(\Sigma_{i} \gamma_{n}^{i}\right) \cap(\Sigma \mu(\beta) \beta)=c$.

Let $\left\langle\underline{\gamma}_{n}, \cdots, \underline{\beta}_{n-s}\right\rangle$ be a simplex of $K^{\prime}$ in support $c$, occurring in $c$ with coefficient $\lambda$. Let $\mu\left(\beta_{n-s}\right)=\epsilon\left(\gamma_{n}, \cdots, \beta_{n-s}\right) \lambda$. This definition of $\mu\left(\beta_{n-s}\right)$ is clearly what the cap product formula requires, but we must show that is is well defined and that $\Sigma \mu(\beta) \beta$ is a cocycle.

To show that $\mu\left(\beta_{n-s}\right)$ is well defined, we must show that if $\sigma$ and $\tau$ are $s$ simplexes of $K^{\prime}$ lying in $\left(\beta_{n-s}\right)^{*}$, then $\epsilon(\sigma) \lambda(\sigma)=\epsilon(\tau) \lambda(\tau)$. Since $X$ is a normal $n$-circuit, $\partial\left(\beta_{n-s}\right)^{*} \cong 1 \mathrm{k}\left(\beta_{n-s} ; X\right)$ is an $(s-1)$-circuit, so $\left(\beta_{n-s}\right)^{*}$ is an $s$-circuit with boundary. Thus it suffices to check the case when $\sigma$ and $\tau$ have a common $(s-1)$-face. Then, since $\partial c=0, \lambda(\sigma)=-\lambda(\tau)$. Checking that $\epsilon(\sigma)=-\epsilon(\tau)$ is an elementary exercise which we leave to the reader. It is also straightforward to check that $\delta(\Sigma \mu(\beta) \beta)=0$. Q.E.D. 
An algebraic argument shows that if $X$ is an $n$-circuit with fundamental class $[X]$, then $[X] \cap \cdot: H^{n-s}(X) \rightarrow H_{s}(X)$ is surjective for all $s$ implies it is an isomorphism for all $s[13]$.

COROLLARY 5.3. Let $X$ be a normal polyhedral n-circuit with fundamental class $[X]$. Then $[X] \cap \cdot: H^{*}(X) \rightarrow H_{*}(X)$ is an isomorphism (i.e., $(X,[X])$ is a Poincare duality space) if and only if every (singular) cycle in $X$ is homologous to a PL cycle whose support is transverse to the intrinsic stratification of $X$.

This corollary generalizes the classical Poincare duality theorem for closed (PL) manifolds, since a closed manifold has but one intrinsic stratum. The way that singularities in a space disturb Poincare duality is explored more fully in [13], using a fascinating spectral sequence of Zeeman.

We have suggested that an appropriate definition of " $P$ is transverse to $Q$ in $X$ ' is that $P$ is transverse to the intrinsic stratification of $Q$ in $X$. In closing, we would like to discuss the more general definition of $P \pitchfork Q$ : that there is a structured cone complex $C$ on $X$ with $Q \sqrt{ } C$ and $P \sqrt{ } C^{*}$. (These definitions are equivalent when $X$ is a manifold and $P$ is proper.)

We will say that $P$ and $Q$ are in general position in $X$ if for each $i$,

$$
\operatorname{dim}\left(P \cap Q \cap X_{i}\right) \leqslant \operatorname{dim}\left(P \cap X_{i}\right)+\operatorname{dim}\left(Q \cap X_{i}\right)-i,
$$

where $X_{i}$ is the intrinsic $i$-stratum of $X$. A pleasant little combinatorial argument shows that $P \pitchfork Q$ implies $P$ and $Q$ are in general position.

Akin has shown that if $P$ and $Q$ are subpolyhedra of $X$, there is an ambient isotopy of $X$ moving $P$ into general position with respect to $Q$. (See [1, p. 471]. For a different proof, which shows that the isotopy can be made arbitrarily small, see [13, p. 98].) In fact, one can show that $P \pitchfork Q$ in $X$ implies that $\left(P \cap X_{i}\right) \pitchfork$ $\left(Q \cap X_{i}\right)$ in $X_{i}$ for each $i$. (The converse is false. For example, let $X$ be the pinched torus, with $P$ and $Q$ two circles not homologous to zero, which intersect only at the singular point of $X$.)

Given $P$ and $Q$ in $X$, it is not always possible to make $P \pitchfork Q$ by an isotopy of of $P$. However, it should always be possible to make $\left(P \cap X_{i}\right) \pitchfork\left(Q \cap X_{i}\right)$ in $X_{i}$ for each $i$ (by an $\epsilon$-ambient isotopy of $X$ ), as suggested by Akin's general position theorem.

\section{REFERENCES}

1. E. Akin, Manifold phenomena in the theory of polyhedra, Trans. Amer. Math. Soc. 143 (1969), 413-473. MR 40 \#6544.

2. (1972), 401-438.

3. M. A. Armstrong, Transversality for polyhedra, Ann. of Math. (2) 86 (1967), 172191. MR 36 \#2158. 
4. M. A. Armstrong and E. C. Zeeman, Transversality for piecewise linear manifolds, Topology 6 (1967), 433-466. MR 36 \#2157.

5. T. Banchoff, Critical points and curvature for embedded polyhedra, J. Differential Geometry 1 (1967), 245-256. MR 37 \#921.

6. G. Birkhoff, Lattice theory, 3rd ed., Amer. Math. Soc. Colloq. Publ., Vol. 25, Amer. Math. Soc., Providence, R. I., 1967. MR 37 \#2638.

7. M. M. Cohen, Simplicial structures and transverse cellularity, Ann. of Math. (2) 85 (1967), 218-245. MR 35 \#1037.

8. - A general theory of relative regular neighborhoods, Trans. Amer. Math. Soc. 136 (1969), 189-229. MR 40 \#2052.

9. M. Cohen and D. Sullivan, On the regular neighborhood of a two-sided submanifold, Topology 9 (1970), 141-147. MR 41 \#9262.

10. E. R. van Kampen, Die Kombinatorische Topologie und die Dualitätssätze, Den Haag (1929).

11. A. Kosiński, Singularities of piecewise linear mappings. I: Mappings into the real line, Bull. Amer. Math. Soc. 68 (1962), 110-114. MR 24 \#A2389.

12. S. Lefschetz, Topology, 2nd ed., Chelsea, New York, 1956.

13. C. McCrory, Poincare duality in spaces with singularities, Ph.D. Thesis, Brandeis University, 1972.

14. H. R. Morton, Joins of polyhedra, Topology 9 (1970), 243-249. MR 41 \#6200.

15. C. P. Rourke and B. J. Sanderson, Block bundles. II: Transversality, Ann. of Math. (2) 87 (1968), 256-278. MR 37 \#2234b.

16. - An embedding without a normal microbundle, Invent. Math 3 (1967), 293-299. MR 36 \#5954.

17. - Decompositions and the relative tubular neighborhood conjecture, Topology 9 (1970), 225-229. MR 41 \#6224.

18. S. Buoncristiano, C. P. Rourke and B. J. Sanderson, A geometric approach to homology theory, London Math. Soc. Lecture Notes Ser., Cambridge (to appear).

19. - Introduction to piecewise linear topology, Ergebnisse der Mathematik 6S, Springer-Verlag, Heidelberg, 1972.

20. H. Seifert and W. Threlfall, Lehrbuch der Topologie, Teubner, Leipzig, 1934; reprint, Chelsea, New York, 1954.

21. J. R. Stallings, Lectures on polyhedral topology. Tata Inst. Fund. Res. Lec. Math., no. 43, Tata Institute of Fundamental Research, Bombay, 1967. MR 38 \#6605.

22. D. A. Stone, Stratified polyhedra, Lecture Notes in Math., no. 252, SpringerVerlag, New York, 1972.

23. E. Hendricks, Triangulation of stratified sets, Ph.D. Thesis, Massachusetts Institute of Technology, Cambridge, Mass., 1973

SCHOOL OF MATHEMATICS, INSTITUTE FOR ADVANCED STUDY, PRINCETON, NEW JERSEY 08540

Current address: Department of Mathematics, Brown University, Providence, Rhode Island 02912 